\title{
In vitro balloon dilatation of mitral valve stenosis: the importance of subvalvar involvement as a cause of mitral valve insufficiency
}

\author{
A S Sadee, A E Becker
}

\begin{abstract}
To investigate the mechanism that increases the orifice area of the mitral valve during balloon dilatation 43 surgically excised intact rheumatic mitral valves were studied. The main pathological features were $(a)$ fibrosis of mitral valve leaflets and commissures (10 valves); (b) fibrosis with calcification of one commissure (eight anterolateral, seven posteromedial); (c) fibrosis with calcification of both commissures (seven valves); and ( $d$ ) predominant involvement of the subvalvar apparatus (11 valves). The valves were assessed by photography and radiography before and after balloon dilatation (balloons up to $38 \mathrm{~mm}$ (bifoil $2 \times 19 \mathrm{~mm}$ ) and piessures up to 4 atmospheres). The valve was dilated in stages under direct visual control by balloons of increasing diameter. Splitting of the fused commissures was the most common mode of widening the orifice. However, the mitral valve leaflets were torn in two fibrotic valves and in six valves with extensive involvement of the subvalvar apparatus. In the fibrotic valves (group $(a)$ ) the tear originated near the valve perimeter, at the thinnest part of the remaining valve leaflet. In the valves with subvalvar involvement splitting started at the apex of spaces between the fused chordal columns and proceeded upward.

Where there is extensive involvement of the subvalvar apparatus in rheumatic mitral valve disease the risk of tearing of the valve leaflets by balloon dilatation is increased and this is likely to predispose to the development of acute valvar insufficiency.
\end{abstract}

Departments of Cardiology and Pathology, University of Amsterdam Academic Medical Center, Amsterdam, The Netherlands A S Sadee

A E Becker

Correspondence to Professor A E Becker Department of Pathology, Academic Medical Center, Meibergdreef 9, $1105 \mathrm{AZ}$ Amsterdam-Zuidoost, The Netherlands.

Accepted for publication 4 January 1991
The first report, by Inoue et al, of transvenous balloon dilatation of mitral valve stenosis in six patients, appeared in $1984 .^{1}$ The patients selected for this new technique did not have calcifications of the mitral valve apparatus or signs of mitral valve insufficiency. Because of good short term results and absence of major complications this procedure was soon used in patients with severe mitral valve stenosis, even those with calcifications and moderately severe mitral valve insufficiency. ${ }^{23}$ At surgical operation Block et al showed that an earlier percutaneous balloon dilatation of the mitral valve had split the fused fibrotic and calcified commissures along their natural planes; ${ }^{4}$ this effect was confirmed by cross sectional echocardiography. ${ }^{5}$ In vitro analysis of the effects of balloon dilatation in surgically excised formalin fixed mitral valves also showed that splitting at the commissures, including those that were heavily calcified, was the prime mechanism. ${ }^{67}$

The initial success of commissurotomy of rheumatic mitral valve stenosis, in which the aim is also to open up fused commissures, was lower in calcified valves and valves with subvalvar involvement. Also restenosis appeared sooner and mitral valve insufficiency became a problem, either because it increased in severity or because it developed as a consequence of the commissurotomy. ${ }^{8-11}$ Because balloon dilatation is basically a comparable procedure it is not surprising that these drawbacks to the technique have recently been recognised in calcified mitral valves and those with subvalvar involvement. ${ }^{512-16}$

In $40-50 \%$ of patients mitral valve insufficiency developed or increased after balloon dilatation; this effect was not foreseen with the echocardiographic grading systems used in earlier studies. ${ }^{21217}$ In most instances the mitral valve regurgitation so created is considered to be of little haemodynamic importance. None the less, acute severe mitral valve insufficiency can occasionally occur. The mitral valves of all of these patients showed extensive subvalvar disease and at surgery paracommissural tears of the valve leaflet were found. ${ }^{1318}$

We found that several groups with rheumatic mitral valve disease can be distinguished on the basis of valve morphology. We investigated whether these groups showed any differences after dilatation of the excised mitral valves and, if so, whether any recommendations can be made about balloon dilatation in vivo.

\section{Patients and methods}

METHODS

We selected 43 mitral valves that were surgically excised because of rheumatic valve stenosis. All selected specimens had valves and subvalvar apparatus removed en bloc. The valves were fixed in $10 \%$ buffered formalin. The valves were classified on the basis of the main pathological feature: (a) a fibrotic group with fibrotic thickening of leaflets and fusion of commissures without calcifications; (b) a group with fibrosis and calcification of either the 
Table Summary of results

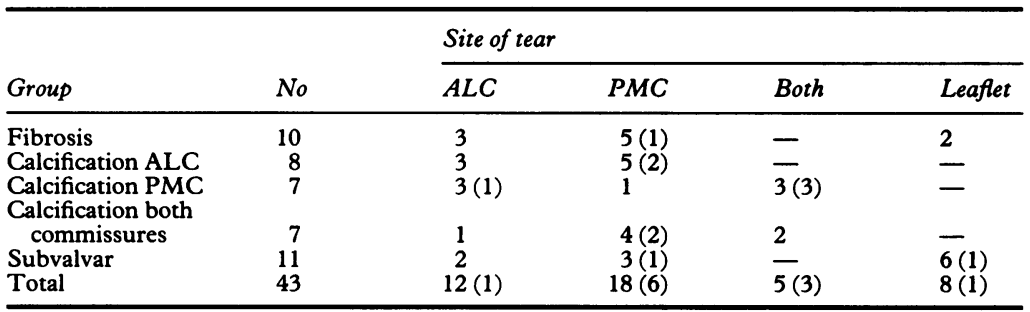

ALC, anterolateral commissure; PMC, posteromedial commissure. Numbers in parentheses are completely disrupted valves.

anterolateral or the posteromedial commissure; (c) a group with calcifications within both fused commissures; $(d)$ a group showing severe subvalvar disease (thickened, shortened, and fused chordae, leading to obliteration of the interchordal spaces) in addition to fibrosis and calcification. In patients in groups $(a)$ to $(c)$ the subvalvar apparatus was not involved or only slightly involved.

Photographs and radiographs were taken before and after dilatation. The degree of calcification was classified as slight, moderate, and severe.

\section{Experimental design}

The mitral valve was dilated with $8 \mathrm{~F} 19 \mathrm{~mm}$ monofoil, 9F $19 \mathrm{~mm}$ trefoil (by placing a ligature around one balloon a circumference was obtained of $24 \mathrm{~mm}$ ), $12 \mathrm{~F} 15 \mathrm{~mm}$ bifoil, 9F $12 \mathrm{~mm}$ trefoil, and $12 \mathrm{~F} 19 \mathrm{~mm}$ bifoil balloon catheters (Schneider). The catheters were attached to a manometer pressure gauge through a Y connection. The deflated balloon

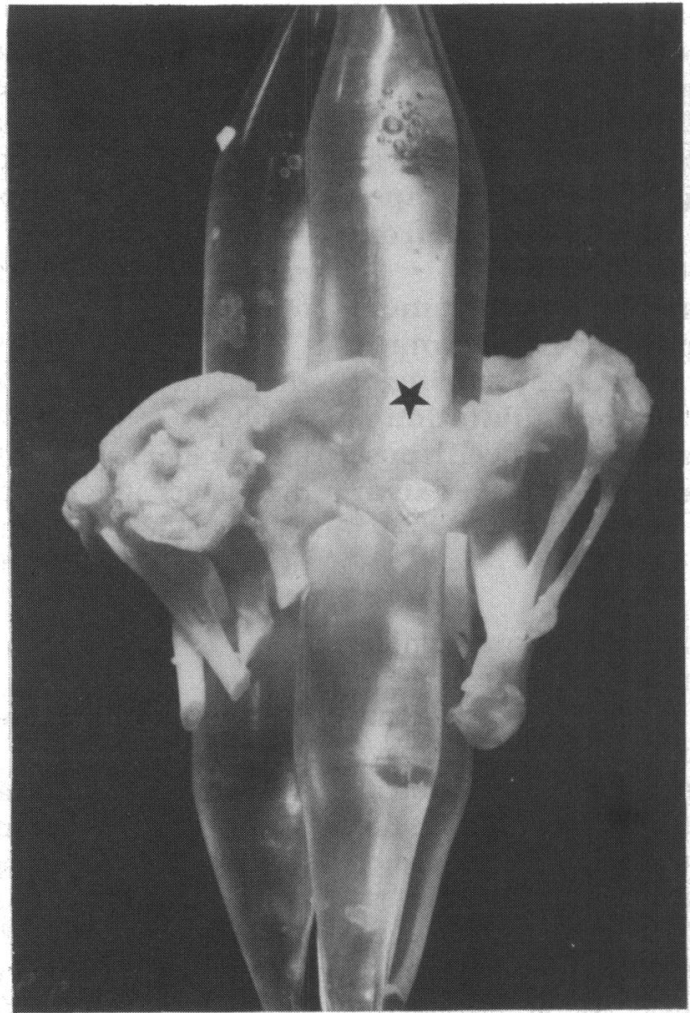

Figure 1 Balloon dilatation of a fibrotic valve. The tear started close to the valve perimeter (asterisk) through a previously fused commissure and proceeded downwards. was introduced from the atrial side of the valve and advanced halfway down the valve.

After inflation of the smallest balloon, at a maximum pressure of 4 atmospheres, larger and larger balloons were used if no effects were seen. The results of this gradual increase in both the pressure and the balloon circumference were under direct visual control.

\section{Results}

The table summarises the results. In 35 out of 43 valves dilatation resulted in a tear, which opened up the fused commissures, both fibrotic and calcified. In 11 of the 43 excised valves the tear led to complete disruption. This event was spotted instantly and its mode of origin was visually identified. These valves, therefore, were not excluded from the evaluation. Ten of these 11 valves were completely disrupted by a continuation of a commissural tear. In the remaining valve there was extensive subvalvar involvement and the tear originated distally within the leaflet and extended upwards into the valve perimeter.

In valves with commissural calcifications no relation was seen between the site of the tear and the site of the calcification. Nor did the degree of calcification affect this outcome. The more severe the calcifications, however, the greater the chance of complete disruption. Seven of the 15 valves with severe calcifications were completely disrupted. Neither of the two moderately calcified valves was disrupted and only one of the remaining five slightly calcified valves. All the tears in the calcified valves were

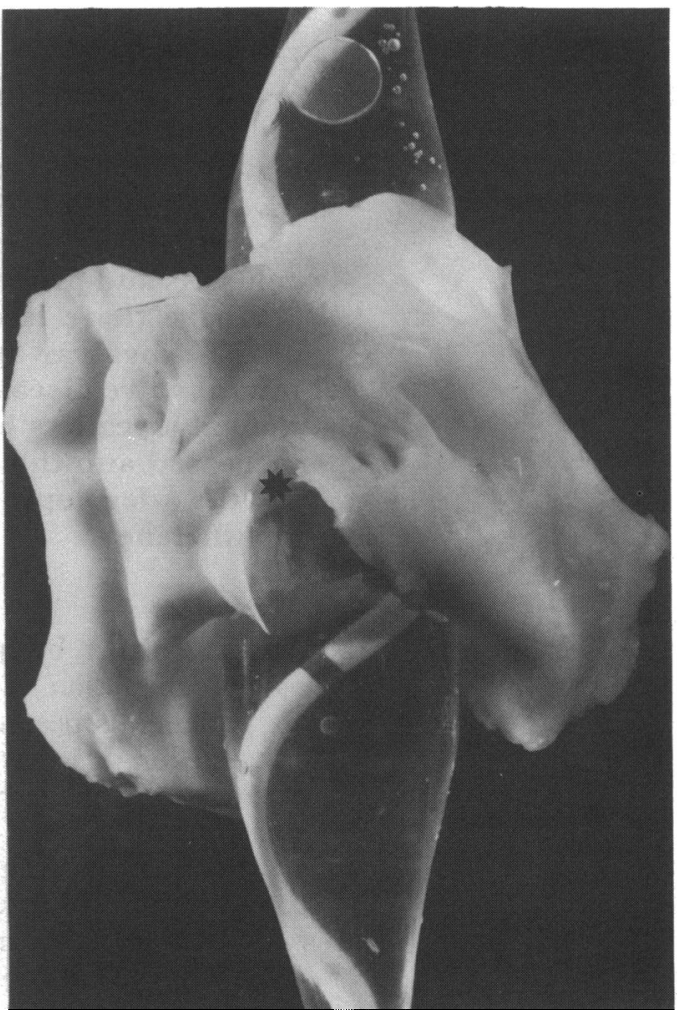

Figure 2 Balloon dilatation of a valve showing severe involvement of the subvalvar apparatus. The tear started at the apical side of the valve and proceeded upwards (asterisk) through the valve leaflet. 
at commissural sites; none of the leaflets was torn.

In eight valves a leaflet was torn-two in valves with fibrosis without calcifications and six in valves with subvalvar involvement. In the fibrosed valves the tear always started to appear close to the valve perimeter at the thinnest part of the mural leaflet of the excised valve (fig 1) and in the group with subvalvar involvement it originated at the apex of spaces between the fused chordal columns and proceeded upward (fig 2).

In the group showing subvalvar involvement the aortic leaflet was torn in five cases and the mural leaflet in one. With one exception, all these unwanted tears occurred between fibrotic columns of fused chordae (fig 2). In one only, the laceration occurred in a severely calcified leaflet. All tears, moreover, originated on the ventricular side of the subvalvar apparatus and proceeded upwards towards the valve perimeter. In one of these complete disruption occurred. Since the calcifications in this group were diffusely dispersed throughout the valvar apparatus the relation, if any, between tears and calcific deposits could not be determined.

\section{Discussion}

Previous studies of in vitro balloon dilatation of the mitral valve showed that the mitral valve orifice increased by splitting of diseased commissures. ${ }^{367}$ Our study confirmed these results. In the earlier studies, however, no lacerations were reported in the leaflets or the subvalvar apparatus but the type of disease affecting the mitral valve was not specified.

The morphology of the rheumatic mitral valve when there is severe involvement of the subvalvar apparatus suggested to us that the mechanism of balloon dilatation might be different and potentially dangerous. The valve leaflets are pulled down like a funnel so that the narrowed and deformed ostium is located well into the ventricle. The fibrosed leaflets contain columns of fused chordae. A balloon positioned and inflated in such an orifice will put pressure upon the walls of the ostium, which will dilate only when the unfortified parts tear. Because the chordae fuse almost randomly, tears can arise in the plane of a commissure or of one of the leaflets. The results of the present study accord with this theory. Of the 11 valves with a severely affected subvalvar apparatus, five showed a commissural tear, as expected, but in six a leaflet was torn. Moreover, each of these leaflet tears originated in the apex of spaces between the fused chordal columns of the affected subvalvar apparatus and extended upwards towards the valve perimeter.

Do the tears in two of the valve leaflets of the fibrotic group contradict our theory? In both instances we saw the tear starting close to the valve perimeter rather than in the apical parts. We believe that the lack of support at the annular level in these two valves was important.
In both only a small rim of the valve leaflet had been excised at the site of the tear. Similarly, the outcome may have been affected by the fact that all valves had been fixed in formalin. This may have altered the mechanical properties of the tissues. Nevertheless, the results obtained in earlier in vitro experiments with formalin fixed valves have been shown to mirror those following balloon dilatation in life. ${ }^{367}$

Despite its limitations we believe that our study may have clinical relevance. Involvement of the subvalvar apparatus may increase the risk of laceration of the valve leaflets during balloon dilatation and this will lead to acute mitral valve regurgitation. In future clinical studies should be directed towards echocardiographic examination of the subvalvar apparatus.

1 Inoue K, Owaki T, Nakamura T, Kitamura F, Miyamoto N Clinical application of transvenous mitral commissurotomy by a new balloon catheter. J Thorac Cardiovasc Surg 1984;87:394-402.

2 Palacios IF, Block PC, Brandi S, et al. Percutaneous balloon valvotomy for patients with severe mitral stenosis. Circulation 1987;75:778-84.

3 McKay RG, Lock JE, Safian RD, et al. Balloon dilation of mitral stenosis in adult patients: postmortem and percutaneous mitral valvuloplasty studies. J Am Coll Cardio 1987;9:723-31.

4 Block PC, Palacios IF, Jacobs ML, Fallon JT. Mechanism of percutaneous mitral valvotomy. Am J Cardiol 1987; 59:178-9.

5 Reid CL, McKay CR, Chandraratna PAN, Kawanishi DT Rahimtoola SH. Mechanisms of increase in mitral valve area and influence of anatomic features in double-balloon, catheter balloon valvuloplasty in adults with rheumatic mitral stenosis: a Doppler and two-dimensional echocarmitral stenosis: a Doppler and two-dimension
diographic study. Circulation 1987;76:628-36.

6 Kaplan JD, Isner JM, Karas RH, et al. In vitro analysis of mechanisms of balloon valvuloplasty of stenotic mitra valves. Am J Cardiol 1987;59:318-23.

7 Ribeiro PA, al Zaibag M, Rajendran V, et al. Mechanism of mitral valve area increase by in vitro single and double balloon mitral valvotomy. Am J Cardiol 1988;62:264-9.

8 Hoeksma TD, Wallace RB, Kirklin JW. Closed mitra commissurotomy. Recent results in 291 cases. Am Cardiol 1966;17:825-8.

9 Grantham RN, Daggett WM, Cosimi AB, et al. Transventricular mitral valvulotomy. Analysis of factors influencing operative and late results. Circulation 1974;50(suppl II): $200-11$.

10 Baker C, Hancock WE. Deterioration after mitral valvotomy. Br Heart J 1960;22:281-94.

11 Ellis LB, Singh JB, Morales DD, Harken DE. Fifteen-totwenty-year-study of one thousand patients undergoing twenty-year-study of one thousand patients undergoing
closed mitral valvuloplasty. Circulation 1973;48:357-64.

12 Palacios IF, Block PC, Wilkins GT, Weyman AE. Followup of patients undergoing percutaneous mitral balloon valvotomy. Analysis of factors determining restenosis. Circulation 1989;79:573-9.

13 Vahanian A, Michel PL, Cormier B, et al. Results of percutaneous mitral commissurotomy in 200 patients. $A m$ $J$ Cardiol 1989;63:847-52.

14 Herrmann HC, Wilkins GT, Abascal VM, Weyman AE Block PC, Palacios IF. Percutaneous balloon mitra valvotomy for patients with mitral stenosis. Analysis of factors influencing early results. $J$ Thorac Cardiovasc Sur 1988;96:33-8.

15 Wilkins GT, Weyman AE, Abascal VM, Block PC, Palacios IF. Percutaneous balloon dilatation of the mitral valve: an analysis of echocardiographic variables related to outcome and the 308 .

16 Abascal VM, Wilkins GT, Choong CY, et al. Echocardiographic evaluation of mitral valve structure and funcdiographic evaluation of mitral valve structure and func-
tion in patients followed for at least 6 months after tion in patients followed for at least 6 months after percutaneous balloon miol 1988;12:606-15.

17 Abascal VM, Wilkins GT, Choong CY, Block PC, Palacios IF, Weyman AE. Mitral regurgitation after percutaneous balloon mitral valvuloplasty in adults: evaluation by pulsed Doppler echocardiography. J Am Coll Cardio 1988;11:257-63.

18 Cequier A, Bonan R, Crépeau J, Dethy M, Dyrda I, Waters DD. Massive mitral regurgitation caused by tearing of the anterior leaflet during percutaneous mitral balloon valvuloplasty. Am J Med 1988;85:100-3. 